Pacific Journal of Mathematics

MINIMAL DOMAINS AND THEIR BERGMAN KERNEL 


\title{
MINIMAL DOMAINS AND THEIR BERGMAN KERNEL FUNCTION
}

\author{
Michael MASChLER
}

1. Introduction. On certain problems which arise in the theory of conformal and pseudo-conformal transformations. Attempts to generalize the Riemann mapping theorem to the case of multiply connected domains lead to the concept of canonical domains, that is, domains of a special geometric "shape" (for example, the entire plane slit along parallel rectilinear lines), onto which any other domain can be mapped conformally. The existence of such mappings, though interesting in itself, is less useful than the Riemann mapping theorem, because the circle has many properties other canonical domains do not possess. Moreover, this situation is worse when one passes to the space of several complex variables. In fact, even in the case of a simply connected domain, a complete set of canonical domains having a well defined geometric shape is not known. If we return to the case of a plane domain and note that the existence of canonical domains can be proved by considering certain extremum problems, we are led to the conclusion that perhaps it is worthwhile to introduce other types of canonical domains, defined by a simpler extremum problem rather than by geometric shape. Indeed, two important types of such domains were investigated by S. Bergman: The minimal domains and the representative domains. (Bergman [2, pp. 27-42]). The shape of these domains (for definition, see $\S 2,6$.) is in general complicated, often they are situated on nonunivalent Riemann hypersurfaces, but they possess many properties which enable us to deduce interesting results in the theory of pseudoconformal transformations.

In the last century, various domain-functions were introduced and applied successfully to all branches of analysis. It proved useful to consider two types of problems: 1. Given some information on a domain-to find estimates for one of its domain functions. 2. Given properties of a domain function belonging to some domain-to obtain information about the domain itself. Theorems of these types lead, for instance, to distortion theorems and to solutions of various extremum

Received February 19, 1955 and in revised form July 12, 1955. Some of the results of this paper were presented in the abstracts of: Bull. Amer. Math. Soc., 60 (1954), 394, Proc. Intern. Math. Congress, Amsterdam, 1954, p. 139, and W. T. Martin, Report on the analysis seminar, Bull. Amer. Math. Soc. 62, (1956), pp. 79-101. This paper is part of the paper "Domain functions and conformal mappings with applications to extremal problems", to be submitted as a thesis for the Ph. D. degree at the Hebrew University, Jerusalem. 
problems.

In the present paper we consider the Bergman kernel function $K_{D}(z, \bar{\zeta})$ of a minimal domain $D$ with a center $t$. We start our investigation by proving that $K_{D}(z, \bar{t})=$ constant for $z \in D$ (§3). Only minimal domains have this property and this fact enables us to deduce various properties of minimal domains and analytic functions defined in such domains $(\S \S 3,4)$. These properties are generalizations of theorems on circles and they are valid only for minimal domains.

It is interesting to note that while most known applications of the kernel function to the theory of pseudo-conformal mapping use properties of the function $K_{D}(z, \bar{z})$, the theorem mentioned above deals with the function $K_{D}(z, \bar{t})$ where the arguments are not necessarily the same. It seems that this fact throws some light on the connection between mapping of domains and a metric defined in a domain which is invariant under pseudo-conformal transformations (Bergman's metric) (see also $\S 6)$.

In $\S 2$ the possibility of a mapping of a domain onto a minimal domain is considered, then in $\S 5$ it is proved that this mapping is unique up to a transformation with constant Jacobian-this justifies the term "canonical domains". Considering the case of mapping of a minimal domain onto another minimal domain (so that the centers do not correspond), we arrive at a distortion theorem. Section 6 is devoted to the class $S$ of domains which are simultaneously minimal and representative with the same center. We define a domain function $J_{\Delta}(w, \bar{\omega})$ and prove that if a domain $\Delta$ can be mapped onto a domain of the class $S$, then $J_{\Delta}(w, \bar{\tau})=$ constant for $w \in \Delta$, where $\tau$ is the inverse image of the center. The function $J_{\Delta}(w, \bar{w})$, for an arbitrary domain $\Delta$, but with identified arguments, is known to be an invariant under pseudo-conformal transformation.

REMARK. The theorems are stated for domains in the space of $n$ complex variables, $n=1,2,3, \cdots<\infty$. We use both notations $z$ and $\left(z_{1}, z_{2}, \cdots, z_{n}\right)$ to indicate a point in this space. In certain cases we apply theorems that were originally published only for $n=1,2$. In these cases the extension to $n>2$ is trivial. In order to state our theories in more generality we found it useful to consider non-univalent domains and certain kinds of domains in which points with different coordinates are considered identified. This meant a slight generalization of the concept of pseudo-conformal transformations (see $\S 2$ ). The definition of the kernel function and known theorems we utilize in this paper can be easily generalized to such domains.

\section{Preliminary remarks. The existence of a mapping of a domain}


onto a minimal domain. In the present paper we assume that each domain we deal with can be mapped pseudo-conformally onto a bounded univalent domain in the space of $n$ complex variables, $(n=1,2,3, \cdots)$. By a pseudo-conformal mapping of a domain $D$ onto a domain $\Delta$ we mean a one-to-one mapping which, except in a denumerable number of analytic segments of manifolds of complex dimensions $\leqq n-1$, can be described locally in the form

$$
w_{k}=w_{k}(z), \quad k=1,2, \cdots, n, z \in D, z \equiv\left(z_{1}, z_{2}, \cdots, z_{n}\right)
$$

where $w_{k}(z)$ are analytic functions with a non-vanishing Jacobian. We allow $w_{k}(z)$ to be multi-valued functions provided that $\partial\left(w_{1}, w_{2}, \cdots, w_{n}\right)$ / $\partial\left(z_{1}, z_{2}, \cdots, z_{n}\right)$ is a single-valued meromorphic function in its domain of definition. In such case we "identify" in $\Delta$ points which correspond to the same point of $D$. A regular function in $\Delta$ must have, by definition, the same value at the identified points.

It is known that such domains possess a Bergman kernel function

$$
K_{D}(z, \bar{t}) \quad(z, t \in D)
$$

(see Bergman [1, p. 30], [3, p. 24, 37], also (2.2)).

If $D$ is a bounded univalent domain then $K_{D}(z, \bar{t})$ is a regular function in $z$ and $\bar{t}$ and $K_{D}(z, \bar{z})>0,(z, t \in D)$; however, in general this is not true. Indeed, let $\Delta$ be a domain obtained from $D$ by the pseudoconformal transformation (2.1), then the relation

$$
K_{D}(z, \bar{t})=\left.K_{\Delta}(w(z), \bar{w}(t)) \begin{gathered}
\partial\left(w_{1}, \cdots, w_{n}\right) \partial\left(w_{1}, \cdots, w_{n}\right) \\
\partial\left(z_{1}, \cdots, z_{n}\right)
\end{gathered}\right|_{z=t}
$$

holds for $z, t \in D$; where $w \equiv\left(w_{1}, \cdots, w_{n}\right)$ (Bergman [3, p. 33], [1, p. 51]).

Hence, if $\Delta$ is a bounded univalent domain and $K_{\Delta}\left(w\left(z^{*}\right), w\left(t^{*}\right)\right) \neq 0$ where $z^{*}$ or $t^{*}$ (or both) is a point on a branch manifold of $D$ - the type of singularity of $K_{D}\left(z^{*}, \bar{t}^{*}\right)$ is determined by the Jacobian of the transformation in (2.2). If for $z=z^{*}$ the Jacobian exists and is equal to zero, then $K_{D}\left(z^{*}, \bar{z}^{*}\right)=0$.

We wish, however, to stress that it may well happen that $z=z^{*}$ or $t=t^{*}\left(\right.$ or both) is a point on a branch manifold of $D$ but $\left.K_{\Delta}\left(w\left(z^{*}\right), \overline{w\left(t^{*}\right.}\right)\right)=0$ in such a way that as $z \rightarrow z^{*}, t \rightarrow t^{*}$, the kernel function $K_{D}(z, \bar{t})$ will tend to a finite value (which, by definition, is $K_{D}\left(z^{*}, \bar{t}^{*}\right)$ ).

Definition. A domain $D$ is called a minimal domain with respect to a point $t(t \in D)$ as center if $t$ is not a point on a branch manifold of $D$ and if any pseudo-conformal transformation $w_{k}=w_{k}(z), k=1,2, \cdots, n$ which satisfies 


$$
\left.\begin{gathered}
\partial\left(w_{1}, \cdots, w_{n}\right) \\
\partial\left(z_{1}, \cdots, z_{n}\right)
\end{gathered}\right|_{z=t}=1
$$

maps $D$ onto a domain whose volume is not less than the volume of $D$.

REMARK. Thus, since we are dealing only with a special type of domains, the volume of a minimal domain is finite.

THeOREM 2.1. Any domain $D$ (of the type mentioned at the beginning of this section) can be mapped pseudo-conformally onto a minimal domain with center at the origin by a transformation (2.1) which satisfies

$$
w_{k}(t)=0,(k=1,2, \cdots, n) ;\left.\begin{gathered}
\partial\left(w_{1}, \cdots, w_{n}\right) \\
\partial\left(z_{1}, \cdots, z_{n}\right)
\end{gathered}\right|_{z=t}=1,
$$

where $t$ is any fixed point of $D$ which does not lie on a branch manifold.

Proof. This theorem was stated for special domains; however, essentially, the the proof is the same for the general case. S. Bergman showed ([2 p. 39] see also Garabedian [5]) that if such a mapping exists, then

$$
\begin{aligned}
& K_{D}(z, \bar{t}) \\
& K_{D}(t, t)
\end{aligned}=\begin{aligned}
& \partial\left(w_{1}, \cdots, w_{n}\right) \\
& \partial\left(z_{1}, \cdots, z_{n}\right)
\end{aligned}
$$

and if there exists a pseudo-conformal transformation which satisfies (2.3) and (2.4) then this transformation maps $D$ onto a minimal domain with the origin as center. The volume $V$ of the minimal domain is

$$
V=\begin{gathered}
1 \\
K_{D}(t, t)
\end{gathered} \text {. }
$$

It remains to be shown that there exists always a pseudo-conformal transformation satisfying (2.3) and (2.4). This is trivial for $n=1$, and for $n>1$ it was proved for domains satisfying special conditions, even with the condition that the transformations (2.1) will be of the type

$$
w_{1}=w_{1}(z), \quad w_{k}=z_{k}-t_{k}, \quad k=2,3, \cdots n .
$$

Among such domains are, for instance, domains of the form $D=B_{1} \cup$ $B_{m} \cup \cdots \cup B_{m}$ where $B_{i},(i=1,2, \cdots, m)$ are polycylinders $\left\{\left|z_{k}-a_{k}^{i}\right|<r^{i}\right.$, $k=1,2, \cdots, n\}$ (S. Bergman [2 p. 38], Schiffer [7]). The existence of a mapping of a general domain $D$ onto a minimal domain now follows directly. We map $D$ onto a bounded schlicht domain $D^{*}$ in the $\zeta$-space by a pseudo-conformal transformation satisfying (2.3). This is possible since $t$ is not a point on a branch manifold. Since any point of $D^{*}$ can be connected to the origin by a chain of a finite number of poly- 
cylinders, we can define by the method of analytic continuation ${ }^{1}$ a function $w_{1}=w_{1}(\zeta)$ regular in $\zeta \in D^{*}\left(\zeta \equiv\left(\zeta_{1}, \zeta_{2}, \cdots, \zeta_{n}\right)\right)$ such that

$$
\frac{\partial w_{1}}{\partial \zeta_{1}}=\frac{K_{D^{*}}(\zeta, 0)}{K_{D^{*}}(0,0)} ; w_{1}(0)=0
$$

The transformation $w_{1}=w_{1}(\zeta), w_{k}=\zeta_{k}, k=2,3, \cdots, n$ maps $D^{*}$ onto a minimal domain $\Delta$ with the origin as center, and the transformation from $D$ onto $\Delta$ satisfies (2.3).

A minimal domain is, in general, not univalent. In fact, by an unpublished result of $M$. Schiffer, in the case $n=1$, except for a trivial case, multiply connected minimal domains are not univalent domains without identified points (see also Kufareff [6])". The trivial case is a circle punctured at isolated points where the center of the circle is the center of this minimal domain (Bergman [3 p. 24]). In the case of several complex variables there are more types of univalent minimal domains. For $n=2$, sufficient conditions for the existence of a mapping of a univalent domain onto a minimal univalent domain were given by $M$. Schiffer [7].

3. Minimal domains and their Bergman kernel function. Minimal domains can be identified by the behavior of their (Bergman) kernel function and this in turn may help us to find more of their properties.

Theorem 3.1. A necessary and sufficient condition for a domain $D$ to be a minimal domain with center at a point $t$ (where $t$ does not lie on a branch manifold) is

$$
K_{D}(z, \bar{t}) \equiv \text { constant }
$$

for $z \in D$.

The value of the constant is $1 / V$, where $V$ is the volume of $D$.

Proof. If $D$ is a minimal domain in the $z$-space then the transformation $w_{k}=z_{k}-t_{k}, k=1,2, \cdots, n$ maps it onto a minimal domain $\Delta$ in the $w$-space with center at the origin. This transformation has a constant Jacobian and it also satisfies (2.3), hence by (2.4) and (2.5)

$$
K_{D}(z, \bar{t}) \equiv K_{D}(t, \bar{t})=\frac{1}{V} .
$$

On the other hand, if for a domain $D$ and a fixed $t \in D$, we have $K_{D}(z, \bar{t})=$ const. for $z \in D$ then $K_{D}(z, \bar{t})=K_{D}(t, \bar{t})>0$, since $t$ is not on a

1 Of course, since in general $w_{1}(\zeta)$ is not single-valued, there are infinitely many minimal domains obtained in this way. This depends on our choice of "cutting" $D^{*}$ to make it simply connected and "pasting back" the points in the image domain.

${ }^{2}$ Kufareff $[\mathbf{6}]$ considers the minimal domain for the ring when the class of functions consists of regular functions having a single-valued integral, 
branch manifold. Here, the transformation $w_{k}=z_{h}-t_{k}$ satisfies (2.3) and (2.4), therefore it maps $D$ onto a minimal domain which is congruent to $D$.

THeOREm 3.2. If $D$ is a domain such that for a fixed point $t \in D$, which does not lie on a branch manifold of $D$, we have $K_{D}(t, \bar{t})=1 / V$, where $V$ is the volume of $D$, then $D$ is a minimal domain with $t$ as center.

Proof. From Theorem 2.1 it follows that $D$ can be mapped onto a minimal domain $\Delta$ by a pseudo-conformal transformation satisfying (2.3). From (2.5) it follows that $\operatorname{vol} D=\operatorname{vol} \Delta$, hence, by definition, $D$ is a minimal domain.

REMARK. It follows from these theorems that domains such as the hypersphere $\left[\left|z_{1}\right|^{2}+\left|z_{3}\right|^{2}+\cdots+\left|z_{n}\right|^{2}<r^{2}\right]$, the polycylinder $\left[\left|z_{1}\right|<r_{1},\left|z_{2}\right|<r_{2}\right.$, $\left.\cdots,\left|z_{n}\right|<r_{n}\right]$ and even the more general Reinhardt circular domains (with center at the origin) are minimal domains with center at the origin, since their kernel function was computed and was shown to satisfy the condition of Theorem 3.1 (or Theorem 3.2) (see Bergman [4]).

Using the reproducing property of the kernel function, one obtains immediately the following result, which is a generalization of a well known theorem about a circle.

THEOREM 3.3. Let $D$ be a minimal domain with $t$ as center. Let $f(z)$ be any function of the class ${ }^{3} \mathscr{L}^{2}(D)$, then

$$
f(t)=\frac{1}{V} \cdot \int_{D} f(z) d \omega,
$$

where $V$ is the volume of $D$ and $d \omega$ is the volume element. Only minimal domains (where $t$ does not lie on a branch manifold) have this property. (Compare also Schiffer [7] for the case of univalent domains).

Proof. If $D$ is a minimal domain, then from Theorem 1 we obtain

$$
f(t)=\int_{D} K_{D}(t, \bar{z}) f(z) d \omega_{z}=\frac{1}{V} \int_{D} f(z) d \omega .
$$

On the other hand, if $D$ is a domain of finite volume such that for each function in $\mathscr{C}^{2}(D)$ (3.2) holds then (3.2) holds in particular for $f(z)=K_{D}(z, \bar{t})$ (see Bergman [3 p. 22-23]). Hence

3 That is $f(z)$ is a regular function after transplanting its values into the bounded univalent domain which corresponds to $D$, and $\int_{D}|f(z)|^{2} d \omega<\infty$. All integrals are taken in the Lebesgue sense, 


$$
1=\int_{D} K_{D}(z, \bar{t}) d \omega_{z}=V \cdot K_{D}(t, \ddot{t}),
$$

and from Theorem 3.2 it follows that $D$ is a minimal domain.

\section{Geometric properties of minimal domains.}

THEOREM 4.1. If $D$ is a minimal domain with the origin as center then so is $\lambda D$ (that is, the aggregate of all points of the form $\lambda z, z \in D$ ) where $\lambda$ is a complex number, $\lambda \neq 0$.

Proof. The domain $\Delta=\lambda D$ is obtained from $D$ by the transformation $w_{k}=\lambda z_{k}, k=1,2, \cdots, n$. From (2.2) it follows that $K_{p}(0,0)=K_{\lrcorner}(0,0)|\lambda|^{2 n}$ and by Theorem $3 \Delta$ is a minimal domain with center at the origin, since $K_{\Delta}(0,0)=1 /\left(|\lambda|^{2 n} \cdot V_{D}\right)=1 / V_{\perp}$.

Theorem 4.2. A minimal domain $D$ cannot have more than one center, provided that for any two different points $t^{(1)}$ and $t^{(2)}$ in $D$ (not on a branch manifold) there exists a function $f(z) \equiv f\left(z ; t^{(1)}, t^{(2)}\right)$ in $\mathscr{L}^{2}(D)$ which assumes different values at $t^{(1)}$ and $t^{(2)}$.

Proof. If $t^{(1)}$ and $t^{(2)}$ are two centers of a minimal domain $D, t^{(1)} \neq t^{(2)}$, then from Theorem 3.3 it follows that

$$
\frac{1}{V} \int_{D} f(z) d \omega=f\left(t^{(1)}\right)=f\left(t^{(2)}\right)
$$

for every function $f(z) \in \mathscr{L}^{2}(D)$, a contradiction.

REMARK. The property of a domain $D$ to have a function $f(z) \in \mathscr{L}^{2}(D)$ such that $f\left(t^{(1)}\right) \neq f\left(t^{(2)}\right)$ when $t^{(1)}$ and $t^{(2)}$ are any given different points (not on a branch manifold), certainly holds for bounded domains, hence also for domains that can be mapped pseudo-conformally onto them; that is, for all domains considered in this paper.

THEOREM 4.3. A product domain $D$ is a minimal domain if and only if its components are minimal domains. The center of $D$ is the product of the centers of the components.

Proof. If $D=D_{1} \times D_{2} \times \cdots \times D_{s}$ and $D_{i}$ are minimal domains with centers $t^{(i)}(i=1,2, \cdots, s)$ then, since

$$
\begin{gathered}
K_{D}(z, \bar{t})=K_{D_{1}}\left(z^{(1)}, \bar{t}^{(1)}\right) \cdot K_{D_{2}}\left(z^{(2)}, \bar{t}^{(2)}\right) \cdots K_{D_{s}}\left(z^{(s)}, \bar{t}^{(s)}\right), \\
z^{(i)} \in D_{i}, \quad z=\left(z^{(1)}, z^{(2)}, \cdots, z^{(s)}\right), \quad t=\left(t^{(1)}, t^{(2)}, \cdots, t^{(s)}\right),
\end{gathered}
$$


(see Springer [8 p. 414]), it follows from Theorem 3.1 that $K_{D}(z, \bar{t})=$ const., $z \in D$. Therefore $D$ is a minimal domain.

The converse is also true. Let $D$ be a minimal domain, then

$$
\underset{\operatorname{vol} D}{1}=K_{D}(t, \bar{t})=K_{D_{1}}\left(t^{(1)}, \bar{t}^{(1)}\right) \cdot K_{D_{2}}\left(t^{(2)}, \bar{t}^{(2)}\right) \cdots K_{D_{s}}\left(t^{(s)}, \bar{t}^{(s)}\right) \text {. }
$$

Since $1 / K_{D_{i}}\left(t^{(i)}, \bar{t}^{(i)}\right)$ is the volume of a minimal domain generated from $D_{i}$ and the point $t^{(i)}$ in $D_{\iota}$ (see 2.5), it follows that

$$
K_{D_{i}}\left(t^{(i)}, \bar{t}^{(i)}\right) \geqq\left(\operatorname{vol} D_{1}\right)^{-1}, \quad i=1,2, \cdots, s .
$$

The equality holds only if $D_{i}$ is a minimal domain with center at $t^{(i)}$. Thus

$$
\begin{gathered}
1 \\
\operatorname{vol} D
\end{gathered} \frac{1}{\operatorname{vol} D_{1}} \cdot \frac{1}{\operatorname{vol} D_{2}} \cdot \cdots \cdot \operatorname{vol} D_{\mathrm{s}}=\begin{gathered}
1 \\
\operatorname{vol} D
\end{gathered}
$$

and the theorem is proved.

5. Transformation of a minimal domain onto a minimal domain. Theorem 2.1. assures us that any domain $D$ can be mapped onto a minimal domain by a pseudo-conformal transformation which is normalized at a point $t \in D$ (the inverse image of the center, and thus does not lie on a branch manifold). There are, in general, infinitely many such transformations which transform $D$ onto different minimal domains under the same conditions. However, we can say that up to a transformation with a constant Jacobian these transformations are the same. More exactly, we have the following.

THEOREM 5.1. Any pseudo-conformal transformation (2.1) which satisfies

$$
w_{k}(0)=0,(k=1,2, \cdots, n) \quad \text { and } \quad \begin{aligned}
& \partial\left(w_{1}, \cdots, w_{n}\right)=\text { const } . \\
& \partial\left(z_{1}, \cdots, z_{n}\right)
\end{aligned}
$$

for every $z \in \Delta_{1}$ where the Jacobian exists, maps the minimal domain $\Delta_{1}$ with center at the origin onto a minimal domain $\Delta_{2}$ with the center at the origin. Conversely any pseudo-conformal transformation (2.1) which maps a minimal domain $\Delta_{1}$ with center at the origin onto a minimal domain with center at the origin such that the centers correspond to each other must satisfy (5.1) whenever the Jacobian exists.

The proof is an immediate consequence of (2.2) and Theorem 3.1. It is of interest to study also transformation of one minimal domain onto another in which the centers do not correspond. From Theorem 4.1 
it follows that we can limit ourselves to minimal domains of equal volume. It is to be expected that properties of transformation of this kind are closely connected with distortion theorems.

THEOREM 5.2. Let a minimal domain 4 with center at the origin be an image of a minimal domain $D$ with center at the origin under a pseudo-conformal transformation (2.1) such that $w_{k}(t)=0, k=1,2, \cdots, n$ and $t \neq 0$. Let us assume also that the two domains have the same volume, then

$$
\left|\begin{array}{c}
\partial\left(w_{1}, \cdots, w_{n}\right) \\
\partial\left(z_{1}, \cdots, z_{n}\right)
\end{array}\right|_{z=t} \mid>1
$$

Lemma. If $D^{*}$ is a minimal domain with center $\zeta$ then

$$
\min _{z \in D} K_{D^{*}}(z, \bar{z})=K_{D^{*}}(\zeta, \bar{\zeta})
$$

for any $z$ which does not lie on a branch manifold and the minimum is achieved only if $z=\zeta$.

Proof. Indeed, we can map $D^{*}$ onto a minimal domain $\Delta^{*}$ with the normalization of Theorem 2.1 at the point $z, z \neq \zeta$. Since $D$ has only one center (see Theorem 4.2) we have vol $D^{*}>$ vol $\Delta^{*}$; on the other hand

$$
\operatorname{vol} D^{*}=\frac{1}{K_{D^{*}}(\zeta, \bar{\zeta})}, \quad \operatorname{vol} \Delta^{*}=\frac{1}{K_{D^{*}}(z, \bar{z})},
$$

hence the lemma is proved.

Proof of Theorem 5.2. By (2.2) we have

$$
K_{D}(t, \bar{t})=\left.K_{\Delta}(0,0) \cdot\left|\begin{array}{c}
\partial\left(w_{1}, \cdots, w_{n}\right) \\
\partial\left(z_{1}, \cdots, z_{n}\right)
\end{array}\right|_{z=t}\right|^{2},
$$

and by the lemma $K_{D}(t, \bar{t})>K_{D}(0,0)=1 / \operatorname{vol} D$; on the other hand $K_{\perp}(0,0)=$ $1 /$ vol $\Delta=1 /$ vol $D$, hence the theorem is proved.

EXAMPLE'. Let $D$ be a domain in the $z$-plane. Let $K_{D}(z, \bar{z})=$ constant on a line $\gamma$ in $D$. We chose a point $A$ on $\gamma$ and map $D$ onto a minimal domain $\Delta_{A}$ by a conformal transformation $w=f_{A}(z)$ which satisfies $f_{A}(A)=0$ $f_{A}^{\prime}(A)=1$, then the image of an arc $\overparen{A B}$ on $\gamma$ whose length is $s$ lies completely in a circle with radius $s$ about the origin in the $w$-plane.

${ }^{4}$ This example can be generalized to the case $n>1$ by using the concept of $B$-area (see Bergman [1]). 
Proof. Let $C$ be any point on $\widehat{A B}$, which does not lie on a branch manifold. We map $D$ onto a minimal domain $\Delta_{G}$ by a conformal transformation $w=f_{c}(z)$ which satisfies $f_{C}(C)=0, f_{C}^{\prime}(C)=1$. From $K_{D}(A, \bar{A})=$ $K_{D}(C, \bar{C})$ it follows that vol $\Delta_{A}=\operatorname{vol} \Delta_{C}$ (see (2.5)), hence from Theorem 5.2 , and (2.4) we have

$$
1>\left|\left[f_{A}^{\prime}(z)\right]_{z=A}^{-1} \cdot\left[f_{C}^{\prime}(z)\right]_{z=A}\right|=1 \cdot\left|\begin{array}{l}
K_{D}(A, \bar{C}) \\
K_{D}(C, \bar{C})
\end{array}\right| ;
$$

hence $\left|K_{D}(A, \bar{C})\right|<K_{D}(A, \bar{A})$.

On the other hand

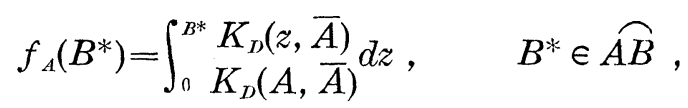

therefore, by chosing the path of integration to be on $\overparen{A B}$ we obtain: $\left|f_{A}\left(B^{*}\right)\right|<s$, from which the required result follows.

6. Minimal domains and representative domains. Attempts to generalize the Riemann mapping theorem to the case of domains in the space of $n$ complex variables lead to various other classes of canonical domains. In this section we shall be interested with the so-called representative domains (Bergman [2 p. 27]):

Let $D$ be a domain in the space of $n$ complex variables $z_{1}, z_{n}, \cdots, z_{n}$. We consider pseudo-conformal transformations of this domain, $w_{k}=$ $w_{k}\left(z_{1}, \cdots, z_{n}\right),(k=1,2, \cdots, n)$, which satisfy

$$
\begin{array}{ll}
w_{k}(t)=t_{k},\left.\quad \frac{\partial w_{k}}{\partial z_{m}}\right|_{z=t}=\delta_{k m}, \quad k, m=1,2, \cdots, n, \\
\delta_{k m}=\text { Kronecker delta },
\end{array}
$$

at a point $t \in D$ (not located on a branch manifold).

Let $^{5}$

$$
M_{D}^{100 \cdots 0}(z, t), M_{D}^{010 \cdots 0}(z, t), \cdots, M_{D}^{000 \cdots 1}(z, t)
$$

be the functions which minimize the integral $\int_{D}|f(z)|^{2} d \omega$ under the conditions

$$
f(z) \in \mathscr{L}^{2}(D), \quad f(t)=0,\left.\frac{\partial f}{\partial z_{k}}\right|_{z=t}=\hat{\delta}_{k l}, \quad l=1, \cdots, n
$$

for $k=1,2, \cdots, n$, respectively.

5 The number of upper indices is $n$. 
Let $M_{J}^{\prime \prime}(z, t)$ be the function which minimizes the same integral under the conditions

$$
f(z) \in \mathscr{L}^{2}(D), f(t)=1
$$

The transformation

$$
\begin{gathered}
w_{1}=\frac{M_{D}^{10 \cdots 0}(z, t)}{M_{D}^{0}(z, t)}+t_{1}, \quad w_{2}=\frac{M_{D}^{01 \cdots 0}(z, t)}{M_{D}^{0}(z, t)}+t_{2}, \cdots, \\
w_{n}=\frac{M_{D}^{00 \cdots 1}(z, t)}{M_{D}^{0}(z, t)}+t_{n}
\end{gathered}
$$

satisfies (6.1) and transforms $D$ onto a domain $\Delta_{t}$. This $\Delta_{t}$ is called a representative domain having $t$ as center.

In the following we shall make use of some properties of representative domains which are proved in Bergman, [2 p. 27-33].

1. A necessary and sufficient condition that two domains can be mapped pseudo-conformally onto each other by a trausformation of the type (6.1) is that they have the same representative domain obtained by (6.2).

2. Equations (6.2) can be expressed in terms of the kernel function $K_{D}(z, \bar{\zeta})$ and its derivative, as follows:

$$
M_{D}^{0}(z, t)=\frac{K_{D}(z, \bar{t})}{K_{D}(t, \bar{t})} \quad(\text { see } \S 2) .
$$

Let $\mathbf{M}$ be the $(n+1) \times(n+1)$ matrix

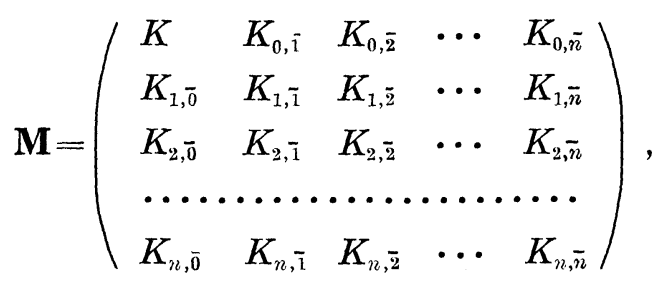

where

$$
K_{i, \bar{j}}=\left.\frac{\partial^{2} K_{D}(z, \bar{\zeta})}{\partial z_{i} \partial \bar{\zeta}_{j}}\right|_{z=t, \bar{\zeta}=\bar{t}} \cdot
$$

Let

$$
K_{0, \bar{i}}(z, \bar{t})=\left.\frac{\partial K_{n}(z, \bar{\zeta})}{\partial \bar{\zeta}_{j}}\right|_{\bar{\zeta}=t},
$$

then 


$$
\begin{aligned}
& \begin{array}{lllll}
0 & K(z, \bar{t}) & K_{0, \mathrm{i}}(\boldsymbol{z}, \bar{t}) & \cdots & K_{0, \bar{n}}(z, \bar{t})
\end{array} \\
& 0 \\
& \begin{array}{l}
0 \\
i_{1} \\
i_{2} \\
\vdots
\end{array} \\
& M_{D}^{i_{1}, i_{2}, \ldots, i_{n}}(z, t)=
\end{aligned}
$$

where $|\mathbf{M}|$ is the determinant of the elements of $\mathbf{M}$ (Bergman [1 p. 45]). It is easy to verify from (6.3) and (6.6) that polycylinders, hyperspheres and, in general, Reinhardt circular domains are representative domains with their center of gravity as center (that is, for these domains (6.2) becomes $\left.w_{k}=z_{k}, k=1,2, \cdots, n\right)^{6}$. These domains are also minimal domains with the same center. In general, however, we shall see (Corollary 2), that minimal domains and representative domains are different.

Theorem 6.1. If a domain $D$ is a minimal domain and also a representative domain with the same center $t$, then its kernel function satisfies the identities

$$
\begin{aligned}
& K_{0, \overline{1}}(z, \bar{t})=A_{11}\left(z_{1}-t_{1}\right)+A_{12}\left(z_{2}-t_{2}\right)+\cdots+A_{1 n}\left(z_{n}-t_{n}\right) \\
& K_{0, \overline{2}}(z, \bar{t})=A_{21}\left(z_{1}-t_{1}\right)+A_{22}\left(z_{2}-t_{2}\right)+\cdots+A_{2 n}\left(z_{n}-t_{n}\right) \\
& \quad \ldots \\
& K_{0, \bar{n}}(z, \bar{t})=A_{n 1}\left(z_{1}-t_{1}\right)+A_{n 2}\left(z_{2}-t_{2}\right)+\cdots+A_{n \eta}\left(z_{n}-t_{n}\right)
\end{aligned}
$$

for $z \in D$. Here $A_{i j}$ are constants and $\left|A_{i j}\right| \neq 0$.

Proof. Since $D$ is a minimal domain it follows from (3.1) of Theorem 3.1 and (6.3) that $M_{D}^{0}(z, t)=1$ for $z \in D$. Also $K_{i, 0},(i=1,2, \cdots, n)$ in (6.6) (see also (6.4)) must vanish. But $D$ is also a representative domain with the same center $t$, hence (6.2) becomes $w_{k}=z_{k}$. We obtain

$$
K_{0, \overline{\mathrm{I}}}(z, \bar{t}) \quad K_{0, \overline{2}}(z, \bar{t}) \cdots K_{0, \bar{z}}(z, \bar{t})
$$

$$
z_{k}-t_{k}=(-1)^{k+1}
$$

$\mathbf{M}_{k}^{*}$

$\left|\mathbf{M}^{*}\right|$

$$
k=1,2, \cdots, n
$$

where $\mathbf{M}^{*}$ is obtained from $\mathbf{M}$ by removing the first row and the first column and $\mathbf{M}_{k}^{*}$ is obtained from $\mathbf{M}^{*}$ by removing the kth row. $\left|\mathbf{M}^{*}\right| \neq 0$ because $|\mathbf{M}| \neq 0$ hence $z_{k}-t_{k}$ satisfy the equations (6.7) where $A_{i}$ are obtained from the corresponding elements of $\mathbf{M}^{*}$.

By reversing the arguments of the proof we obtain immediately the converse theorem

${ }^{6}$ In fact, all circular domains are representative domains with respect to their center (Bergman:[2 p. 32]). 
If $D$ is a minimal domain with $t$ as center, and its kernel function satisfies (6.7) with $\left|A_{i}\right| \neq 0$, then $D$ is also a representative domain with the same center.

Corollary. The constants $A_{i, j}$ of (6.7) must satisfy

$$
A_{i j}=A_{j i} .
$$

Proof. From the symmetry property of the kernel function (Bergman [3 p. 23]) $K_{D}(z, \bar{\zeta})=K_{D}(\zeta, \bar{z})$ it follows that

$$
\partial K_{D}(z, \bar{\zeta}) / \partial \bar{\zeta}_{i}=\partial K_{D}(\zeta, \bar{z}) / \partial \zeta_{i} .
$$

From (6.7) we obtain

$$
\left.\frac{\partial}{\partial \bar{\zeta}_{i}} K_{D}\left(t_{1}, \cdots, t_{j-1}, z_{j}, t_{j+1}, \cdots t_{n} ; \bar{\zeta}_{1}, \bar{\zeta}_{2}, \cdots, \zeta_{n}\right)\right|_{\bar{\zeta}=\bar{t}}=A_{i, j}\left(z_{j}-t_{j}\right),
$$

hence

$$
\left.\underset{\partial \zeta_{i}}{\partial} K_{D}\left(\zeta_{1}, \cdots, \zeta_{n} ; \bar{t}_{1}, \bar{t}_{22}, \cdots, \bar{t}_{j-1}, \bar{z}_{j}, \bar{t}_{j+1}, \cdots \bar{t}_{n}\right)\right|_{\zeta=t}=\bar{A}_{i j}\left(z_{j}-t_{j}\right) .
$$

We consider the Taylor expansions of the function

$$
F\left(z_{i}, \bar{\zeta}_{i}\right) \equiv K_{D}\left(t_{1}, \cdots, t_{i-1}, z_{i}, t_{i+1}, \cdots, t_{n} ; \bar{t}_{1}, \cdots, \bar{t}_{j-1}, \bar{\zeta}_{j}, \bar{t}_{j+1}, \cdots, \bar{t}_{n}\right)
$$

around the point $z_{i}=t_{i}$ and around the point $\bar{\zeta}_{j}=\bar{t}_{j}$. Since

$$
\frac{\partial F\left(t_{i}, \bar{\zeta}_{i}\right)}{\partial z_{i}} \cdot\left(z_{i}-t_{i}\right)=\bar{A}_{i j}\left(\bar{\zeta}_{j}-\bar{t}_{j}\right)\left(z_{i}-t_{i}\right)
$$

and

$$
\frac{\partial F\left(z_{i}, \bar{t}_{j}\right)}{\partial \bar{\zeta}_{j}}\left(\bar{\zeta}_{j}-\bar{t}_{j}\right)=A_{j i}\left(z_{i}-t_{i}\right)\left(\bar{\zeta}_{j}-\bar{t}_{j}\right)
$$

we have (6.9).

Let $D$ be a minimal domain in the $z$-space which is also a representative domain with respect to the same center $t$. Let $\Delta$ be the image of $D$ under the pseudo-conformal transformation $w_{h}=w_{k}(z), k=1,2, \cdots, n$; which satisfies

$$
\left.\frac{\partial\left(w_{1}, \cdots, w_{n}\right)}{\partial\left(z_{1}, \cdots, z_{n}\right)}\right|_{z=t}=1
$$

We use the following notation

$$
\tau_{k}=w_{k}(t),(k=1,2, \cdots, n) ; \quad \omega_{k} \equiv \omega_{k}(\zeta)=w_{k}(\zeta),
$$


then, since

$$
\begin{gathered}
\partial\left(w_{1}, \cdots, w_{n}\right) \\
\partial\left(z_{1}, \cdots, z_{n}\right)
\end{gathered}=\frac{K_{\Delta}(\tau, \bar{\tau})}{K_{\Delta}(w, \bar{\tau})} \quad(\text { see }(2.4),
$$

it follows from (2.2) that

$$
K_{D}(z, \bar{\zeta})=K_{\Delta}(w, \bar{\omega}) \frac{K_{\Delta}(\tau, \bar{\tau})}{K_{\Delta}(w, \bar{\tau})} \cdot \frac{K_{\Delta}(\tau, \bar{\tau})}{K_{\Delta}(\tau, \bar{\omega})},
$$

hence, from (6.7)

$$
\begin{aligned}
& A_{i j}=\left.\frac{\partial^{2} K_{D}(z, \bar{\zeta})}{\partial z_{j} \partial \bar{\zeta}_{i}}\right|_{\bar{\zeta}=\bar{t}} \\
& =\sum_{\mu=1}^{n} \sum_{\nu=1}^{n}\left[\begin{array}{ccc}
K_{\Delta}(\tau, \bar{\tau}) & \partial^{2} K_{\Delta}(w, \bar{\tau}) \\
K_{\Delta}(w, \bar{\tau}) & \partial w_{\mu} \partial \bar{\omega}_{\nu}
\end{array}-\begin{array}{c}
K_{\Delta}(\tau, \bar{\tau}) \\
{\left[K_{\Delta}(w, \bar{\tau})\right]^{2}}
\end{array} \cdot \frac{\partial K_{\Delta}(w, \bar{\tau})}{\partial \bar{\omega}_{\nu}}\right. \\
& \left.\cdot \frac{\partial K_{\Delta}(w, \bar{\tau})}{\partial w_{\mu}}\right]\left.\cdot \frac{\partial \omega_{\nu}}{\partial \zeta_{i}}\right|_{\zeta=t} \cdot \frac{\partial w_{\mu}}{\partial z_{j}} ;
\end{aligned}
$$

therefore, from (6.11) and (6.12)

$$
\begin{gathered}
\left|A_{i j}\right|=\frac{\left[K_{\Delta}(\tau, \bar{\tau})\right]^{n+1}}{\left[K_{\Delta}(w, \bar{\tau})\right]^{n+1}}\left|a_{i j}\right|, \\
a_{\iota \jmath}=\frac{\partial^{2} K_{\Delta}(w, \bar{\tau})}{\partial w_{i} \partial \bar{\omega}_{j}}-\frac{1}{K_{\Delta}(w, \bar{\tau})} \frac{\partial K_{\Delta}(w, \bar{\tau})}{\partial w_{i}} \frac{\partial K_{\Delta}(w, \bar{\tau})}{\partial \bar{\omega}_{j}}, \quad i, j=1,2, \cdots, n, \\
\left(\begin{array}{c}
\partial K_{\Delta}(w, \bar{\tau}) \\
\partial \bar{\omega}_{\nu}
\end{array} \frac{\left.\frac{\partial K_{\Delta}(w, \bar{\omega})}{\partial \bar{\omega}_{\nu}}\right|_{\bar{\omega}=\bar{\tau}}}{}\right) .
\end{gathered}
$$

By expanding the determinant $\left|a_{\iota j}\right|$ we obtain

$$
\begin{aligned}
& \left|A_{i j}\right| \cdot \frac{1}{\left[K_{\Delta}(\tau, \bar{\tau})\right]^{n+1}}=J_{\Delta}(w, \bar{\tau}) \\
& \equiv \frac{1}{\left[K_{\Delta}(w, \tau)\right]^{n+2}} \cdot\left|\begin{array}{cccc}
K_{\Delta}(w, \bar{\tau}) & \frac{\partial K_{\Delta}(w, \bar{\tau})}{\partial \bar{\omega}_{1}} & \ldots & \frac{\partial K_{\Delta}(w, \bar{\tau})}{\partial \bar{\omega}_{n}} \\
\frac{\partial K_{\Delta}(w, \bar{\tau})}{\partial w_{1}} & \frac{\partial^{2} K_{\Delta}(w, \bar{\tau})}{\partial w_{1} \partial \bar{\omega}_{1}} & \ldots & \frac{\partial^{2} K_{\Delta}(w, \bar{\tau})}{\partial w_{1} \partial \bar{\omega}_{n}} \\
\vdots & \ldots \ldots \ldots \ldots \ldots & \ldots \ldots \ldots \\
\frac{\partial K_{\Delta}(w, \bar{\tau})}{\partial w_{n}} & \frac{\partial^{2} K_{\Delta}(w, \bar{\tau})}{\partial w_{n} \partial \bar{\omega}_{1}} & \ldots & \frac{\partial^{2} K_{\Delta}(w, \bar{\tau})}{\partial w_{n} \partial \bar{\omega}_{n}}
\end{array}\right| .
\end{aligned}
$$

The left side of (6.13) is constant, hence we have proved the following. 
THEOREM 6.2. If $\Delta$ is a domain in the w-space which is "equivalent" to a domain $D$; that is, $\Delta$ is the image of $D$ under the pseudo-conformal transformation $\left[w_{k}=w_{k}(z), k=1,2, \cdots, n\right], z \in D$, with a non-vanishing Jacobian at $z=t,{ }^{7}$ and if $D$ is a minimal domain and simultaneously $a$ representative domain with a common center at $z=t$, then the right side of (6.13) is a constant for fixed $\tau$-the image of $t$-and $w \in \Delta$.

REMARK. Since $K_{\Delta}(w, \bar{\omega})=K_{\Delta}(\omega, \bar{u})$, it follows that $J_{\Delta}(w, \bar{\omega})=J_{\Delta}(\omega, \bar{u})$, and hence, under the condition of the last theorem, also $J_{\Delta}(\tau, \bar{v})=$ constant for $w \in \Delta$.

CoRollary 1. Let $\Delta$ be a simply connected plane domain the boundary of which consists of more than one point. Such a domain can be mapped onto a circle such that an arbitrary fixed point $\omega$ of $\Delta$ will be mapped onto the center with a non-vanishing derivative there. Since the circle is a minimal domain and also a representative domain with respect to its center it follows that for any such simply connected domain 4

$$
\left[K_{\Delta}(w, \bar{\omega})\right]^{3} \cdot\left|\begin{array}{cc}
K_{\Delta}(w, \bar{\omega}) & \frac{\partial K_{\Delta}(w, \bar{\omega})}{\partial \bar{\omega}} \\
\frac{\partial K_{\Delta}(w, \bar{\omega})}{\partial w} & \frac{\partial^{2} K_{\Delta}(w, \bar{\omega})}{\partial w \partial \bar{\omega}}
\end{array}\right|=\mathrm{constant}
$$

where both $w$ and $\omega$ vary in $\Delta$.

A similar result can be stated for domains which are equivalent to hyperspheres or to polycylinders, because such domains can be mapped onto themselves with an arbitrary fixed point going to the center.

COROLLARY 2. There are representative domains which are not minimal domains with the same center.

Proof: Otherwise, for all domains $\Delta$ the relation $J_{\Delta}(w, \bar{\omega})=$ constant will hold for $w \in \Delta, \omega \in \Delta$, and in particular $J_{\Delta}(w, \bar{w})=$ constant for $w \in \Delta$. But it is known that there are domains for which this is not true. In fact, it was proved by Springer [8] that, in the case $n=2$, for certain Reinhardt-circular-domains $J_{\Delta}(w, \bar{w})$ is not constant ${ }^{8}$.

It is interesting to note that in the particular case $w=\omega$ one can look at the expression $J_{\Delta}(w, \bar{w})$ from a different angle. Indeed, it was proved by S. Bergman that for any domain (which possesses a kernel

7 The normalization (6.11) is unessential because by a similarity transformation $D$ is mapped onto a domain of the same type (see Theorem 4.1).

${ }^{8}$ In $[8]$ the expression $\left(T_{11} T_{2 \overline{2}}-\left|T_{12}\right|^{2}\right) / K_{\Delta}(w, \bar{w})$, where $T_{m \bar{n}}=\partial^{2} \log K_{\Delta}(w, \bar{w}) / \partial w_{m} \partial \bar{w}_{n}$, is used instead of $J_{\Delta}(w, \bar{w})$. These two expressions are equal as was pointed out by $\mathrm{S}$. Bergman ([1, pp. 52, 53]) 
function) the expression $J_{\Delta}(w, \bar{u})$ is invariant under pseudo-conformal transformation $\left(\left[3\right.\right.$, p. 139]). Estimation of $J_{\Delta}(w, \bar{w})$ was used by various authors to obtain distortion theorems $([3, \mathrm{p} .140],[6],[9]$.$) Thus it$ appears that the domain function $J_{\Delta}(w, \bar{\omega})$ where the argument are, in general, different may yield some information about pseudo-conformal properties of the domain $\Delta$. The connection between $J_{\Delta}(w, \bar{\omega})$ and $J_{\Delta}(w, \bar{w})$ is, in a way, the same as the connection between the kernel function $K_{\Delta}(w, \bar{\omega})$ and the invariant metric based on the function $K_{\Delta}(w, \bar{w})$.

\section{REFERENCES}

1. S. Bergman, Sur les fonctions orthogonales de plusieurs variables complexcs avec les applications à la théorie des fonctions analytiques, Intersc. Pub. 1941, and Mém. des Sc. Math., 106, Paris, 1947.

2. - Sur la fonction-noyau d'un domaine et ses applications dans la théorie des transformations pseudo-conformes, Mém. des Sc. Math., 108, Paris, 1948.

3. - The kernel function and conformal mapping, Amer. Math. Soc., New York, 1950.

4. Ueber die Kernfunktion gewisser Reinhardtscher Kreiskörper, Rev. Math. de l'Union Interbalcanique, 2 (1939), 41-43.

5. P. R. Garabedian, A new formalism for functions of several complex variables, J. Analyse Math., 1 (1951), 59-80.

6. P. Kufareff, Ueber das zweifachzusammenhängende Minimalgebiet, Bull. Inst. Math. et Méc., Univ. de Tomsk, 1 (1935-1937), 228-236.

7. M. Schiffer, Sur les domaines minima dans la théorie des transformations pseudoconformes, C. R. Acad. Sci. Paris, 207 (1938), 112-115.

8. G. Springer, Pseudo-conformal transformations onto circular domains, Duke Math. J., 18 (1951), 411-424.

9. J. Stark, On distortion in pseudo-conformal mapping, Pacific J. Math., 6 (1956), 565582.

Stanford University and The Hebrew University, Jerusalem. 


\section{PACIFIC JOURNAL OF MATHEMATICS}

\section{EDITORS}

\author{
H. L. Royden \\ Stanford University \\ Stanford, California \\ E. Hewitt \\ University of Washington \\ Seattle 5 , Washington
}

R. P. Dilworth

California Institute of Technology

Pasadena 4, California

\author{
A. HorN* \\ University of California \\ Los Angeles 24, California
}

\section{ASSOCIATE EDITORS}

\author{
E. F. BECKENBACH \\ C. E. BURGESS \\ H. BUSEMANN \\ H. FEDERER
}

\author{
M. HALL \\ P. R. HALMOS \\ V. GANAPATHY IYER \\ R. D. JAMES
}

\author{
M. S. KNEBELMAN \\ I. NIVEN \\ T. G. OSTROM \\ M. M. SCHIFFER
}

J. J. STOKER

G. SZEKERES

F. WOLF

K. YOSIDA

\section{SPONSORS}

\author{
UNIVERSITY OF BRITISH COLUMBIA \\ CALIFORNIA INSTITUTE OF TECHNOLOGY \\ UNIVERSITY OF CALIFORNIA, BERKELEY \\ UNIVERSITY OF CALIFORNIA, DAVIS \\ UNIVERSITY OF CALIFORNIA, LOS ANGELES \\ UNIVERSITY OF CALIFORNIA, SANTA BARBARA \\ MONTANA STATE UNIVERSITY \\ UNIVERSITY OF NEVADA \\ OREGON STATE COLLEGE \\ UNIVERSITY OF OREGON \\ UNIVERSITY OF SOUTHERN CALIFORNIA
}

STANFORD RESEARCH INSTITUTE

STANFORD UNIVERSITY

UNIVERSITY OF UTAH

WASHINGTON STATE COLLEGE

UNIVERSITY OF WASHINGTON

AMERICAN MATHEMATICAL SOCIETY HUGHES AIRCRAFT COMPANY SHELL DEVELOPMENT COMPANY

Mathematical papers intended for publication in the Pacific Journal of Mathematics should be typewritten (double spaced), and the author should keep a complete copy. Manuscripts may be sent to any of the editors. Manuscripts intended for the outgoing editors should be sent to their successors. All other communications to the editors should be addressed to the managing editor, Alfred Horn at the University of California, Los Angeles 24, California.

50 reprints of each article are furnished free of charge; additional copies may be obtained at cost in multiples of 50 .

The Pacific Journal of Mathematics is published quarterly, in March, June, September, and December. The price per volume (4 numbers) is $\$ 12.00$; single issues, $\$ 3.50$. Back numbers are available. Special price to individual faculty members of supporting institutions and to individual members of the American Mathematical Society: $\$ 4.00$ per volume; single issues, $\$ 1.25$.

Subscriptions, orders for back numbers, and changes of address should be sent to Pacific Journal of Mathematics, c/o University of California Press, Berkeley 4, California.

Printed at Kokusai Bunken Insatsusha (International Academic Printing Co., Ltd.), No. 10, 1-chome, Fujimi-cho, Chiyoda-ku, Tokyo, Japan.

* During the absence of E. G. Straus.

PUBLISHED BY PACIFIC JOURNAL OF MATHEMATICS, A NON-PROFIT CORPORATION COPYRIGHT 1956 BY PACIFIC JOURNAL OF MATHEMATICS 


\section{Pacific Journal of Mathematics}

\section{Vol. 6, No. $3 \quad$ BadMonth, 1956}

Richard Arens and James Eells, Jr., On embedding uniform and topological spaces ............................................ 397

N. Aronszajn and Prom Panitchpakdi, Extension of uniformly continuous transformations and hyperconvex metric spaces .............. 405

Kai Lai Chung and Cyrus Derman, Non-recurrent random walks ........ 441

Harry Herbert Corson, III, On some special systems of equations . . . . . . . . . 449

Charles W. Curtis, On Lie algebras of algebraic linear transformations . . . 453

Isidore Heller, Neighbor relations on the convex of cyclic permutations . . . . 467

Solomon Leader, Convergence topologies for measures and the existence of transition probabilities..................................... 479

D. H. Lehmer, On certain character matrices ...................... 491

Michael Bahir Maschler, Minimal domains and their Bergman kernel function ........................................... 501

Wm. M. Myers, Functionals associated with a continuous transformation ................................... 517

Irving Reiner and Jonathan Dean Swift, Congruence subgroups of matrix groups ....................................... 529

Andrew Sobczyk, Simple families of lines ................... 541

Charles Standish, A class of measure preserving transformations ........ 553 Jeremiah Milton Stark, On distortion in pseudo-conformal mapping ..... 565 